\title{
How do Technical Factors Affect Developers in Mobile Software Ecosystems
}

\author{
Caio Steglich ${ }^{1}$, Sabrina Marczak ${ }^{1}$, Rodrigo Santos ${ }^{2}$, \\ Luiz Guerra $^{1}$, Luiz Mosmann ${ }^{1}$, Cleidson de Souza ${ }^{3}$, \\ Fernando Figueira Filho ${ }^{4}$, Marcelo Perin ${ }^{5}$ \\ ${ }^{1}$ Escola Politécnica, PPGCC - PUCRS \\ Porto Alegre - RS - Brasil \\ ${ }^{2}$ Programa de Pós-Graduação em Informática - UNIRIO \\ Rio de Janeiro - RJ - Brasil \\ ${ }^{3}$ Programa de Pós-Graduação em Ciência da Computação - UFPA \\ Belém - PA - Brasil \\ ${ }^{4}$ Departamento de Informática e Matemática Aplicada - UFRN \\ Natal - RN - Brasil \\ ${ }^{5}$ Pesquisador Independente \\ Porto Alegre - RS - Brasil \\ \{caio.borges, luiz.guerra, luiz.mosmann\}eacad.pucrs.br \\ sabrina.marczak@pucrs.br, rps@uniriotec.br, cleidson.desouza@acm.org \\ fernando@dimap.ufrn.br, mperin25@gmail.com
}

\begin{abstract}
The Software Evolution area brings applications to the Mobile era in which users want to use these applications on their mobile devices. A Mobile Software Ecosystem (MSECO) is the kind of ecosystems in which developers build applications to attend the needs of mobile technologies users (e.g., Android and $i O S)$. Literature explains that the capability to attracting and retaining people (i.e., developers and users) is essencial to MSECO sustainability, i.e., to the MSECO survive along the years. In a previous work, we conducted a literature review that identified 6 factors that may influence developers to participate in an MSECO. In this study, we present a Field Study aiming to understand how these 6 identified factors may have influenced practitioners in real life projects.
\end{abstract}

Resumo. A área de Evolução de Software traz os aplicativos para a era da mobilidade, aonde os usuários desejam usar esses aplicativos em seus dispositivos móveis. Um ecossistema de software móvel (MSECO) é o tipo de ecossistema em que os desenvolvedores criam aplicativos para atender aos usuários de tecnologias móveis (por exemplo, Android e iOS). A literatura explica que é fundamental para a sustentabilidade do MSECO a capacidade de atrair e reter pessoas (ou seja, desenvolvedores e usuários) para sobreviver ao longo dos anos. Em um trabalho anterior, realizou-se uma Revisão de Literatura, na qual foram identificados 6 fatores que podem influenciar os desenvolvedores a participar de um MSECO. Neste estudo, apresenta-se um Estudo de Campo que teve como objetivo identificar como esses 6 fatores influenciaram profissionais da área em projetos reais vivenciados pelos mesmos. 


\section{Introdução}

A sociedade tem evoluído constantemente, principalmente em termos tecnológicos. Visando atender novas demandas das pessoas, soluções de softwares têm migrado mais e mais para plataformas móveis, fazendo com que os Ecossistemas de Software Móvel (do inglês, MSECO) tornem-se cada vez mais presentes, atraindo usuários e desenvolvedores.

Os Ecossistemas de Software (ECOS) são sistemas complexos no quais tem sido produzido soluções em software na atualidade. Estes ECOS são compostos basicamente por um conjunto interno e externo de desenvolvedores, uma comunidade de especialistas de domínio em serviço e uma comunidade de usuários, oferecendo assim, soluções relevantes para satisfazer as necessidades desses usuários [Bosch and Bosch-Sijtsema 2010].

Tipicamente, um ECOS pode ser visualizado por três dimensões [Campbell and Ahmed 2010]: a dimensão técnica, que compreende as tecnologias que compõem o ECOS (e.g., recursos de desenvolvimento, linguagens de programação, etc); a dimensão de negócio, que compreende elementos como as vendas de aplicações, linhas de produção, entre outros; e a dimensão social, que é composta pelas pessoas que participam do ECOS, como, por exemplo, usuários ou desenvolvedores desse ECOS.

ECOS Móvel são ECOS que atuam sobre o contexto móvel [Fontao et al. 2015], ou seja, aqueles que tem como objetivo a produção de aplicações para dispositivos móveis (e.g. smartphones). Esta classe de ECOS possui dois grandes líderes de mercado, o Android e o iOS [Mallinson 2015]. Pela importância que estes ECOS têm, torna-se fundamental o estudo sobre sua sustentabilidade, sendo esta baseada em dois pilares principais: i) a adaptação a novas tecnologias, recursos e tendências e ii) a atração e manutenção de membros na comunidade do ECOS [Dhungana et al. 2010]. Portanto, pesquisas são necessárias para explorar estes dois pilares e identificar o papel da tecnologia, i.e., a dimensão técnica, utilizada no desenvolvimento e sustentabilidade destas aplicações e MSECO como um todo.

Este trabalho tem como objetivo investigar como fatores técnicos podem influenciar os desenvolvedores de um ECOS móvel na escolha e permanência de um determinado ecossistema para atuarem. Para isso, baseado em um mapeamento sistemático da literatura anterior de autoria dos autores deste artigo que identificou esses fatores [Borges et al. 2019], realizou-se um estudo de campo com desenvolvedores de ECOS móvel, que contou com 20 profissionais. Neste estudo, buscou-se que os participantes explicassem, a partir de suas experiências, como os fatores influenciaram suas decisões de participar ou se manter participando em um ECOS móvel. Identificou-se como os 6 fatores advindos da literatura influenciaram os desenvolvedores a participarem em um MSECO.

O restante deste artigo está organizado como segue: a Seção 2 discorre sobre a metodologia utilizada para a condução desta pesquisa, a Seção 3 apresenta os resultados a partir do processo utilizado, a Seção 4 discute o impacto dos resultados obtidos, a Seção 5 explica as limitações desta pesquisa, e a Seção 6 conclui o artigo.

\section{Metodologia de Pesquisa}

Este estudo é baseado em um estudo anterior, um mapeamento sistemático da literatura [Borges et al. 2019], que identificou 29 fatores relacionados às três dimensões de um ECOS. Usou-se aqui os 6 fatores referentes à dimensão técnica, sendo esses: 
- (F1) Recursos técnicos necessários ou desejados pelo desenvolvedor

- (F2) Desempenho do hardware das aplicações

- (F3) Plataforma facilmente configurável

- (F4) Padrões de interface e aparência das aplicações

- (F5) Segurança dos dados

- (F6) Acessibilidade e suporte a diferentes perfis de usuários

\subsection{Estudo de Campo}

Para a realização do estudo de campo sobre os fatores técnicos identificados, vinte desenvolvedores foram convidados para expressarem como tais fatores os influenciaram, tanto para começar quando para continuar participando de um ECOS móvel.

Singer et al. (2008) [Singer et al. 2008] explicam que um estudo de campo visa investigar como praticantes de alguma atividade lidam com a prática ou resolvem problemas dentro de seus respectivos contextos. Portanto, a partir de um estudo de campo e suas respectivas técnicas de coleta e análise de dados, capturou-se evidências para nosso estudo. Ainda, seguindo as recomendações dos mesmos autores [Singer et al. 2008], desenvolvedores foram convidados, sendo selecionados por conveniência e disponibilidade, contanto que cumprissem o requisito de terem experiência com desenvolvimento em tecnologias móvel. Estes desenvolvedores, todos brasileiros, têm colaborado com o MSECO Android (6 dos participantes) ou iOS (5), ou ambos (9). A experiência dos mesmos com estes MSECO varia entre 1 a 9 anos, e os portes das empresas em que trabalham são distintos-pequena (9 desenvolvedores), média (7) ou grande (4).

As entrevistas duraram em média 25 minutos, sendo a menor delas de 20 minutos e a maior de 30 minutos. Todas entrevistas foram realizadas pessoalmente, gravadas com a autorização do participante e transcritas para análise de texto seguindo a técnica de Card Sorting de Spencer [Spencer 2009]. Os resultados são apresentados a seguir, na Seção 3.

\section{Fatores Técnicos e sua Influência sobre os Desenvolvedores}

A partir das entrevistas com os 20 desenvolvedores de ECOS móvel, identificou-se a importância que os mesmos acreditam que cada fator possui. Como resultado, tem-se que a Segurança dos dados, Padrões de interface e Recursos técnicos desejados são considerados os fatores mais importantes para se motivar um desenvolvedor a participar de um MSECO. Fatores como o Desempenho do hardware ganha grande importância para que o desenvolvedor se mantenha no ECOS.

Além disso, os desenvolvedores discorreram sobre como eles veem cada um dos fatores, conforme apresentado a seguir.

\section{(F1) Recursos técnicos desejados pelo desenvolvedor}

Os recursos técnicos foram considerados importantes para começar a participação em um ECOS móvel por 14 desenvolvedores entrevistados, sendo que alguns destes destacam que os recursos técnicos são usados como critério de decisão (D3, D10) para que ingressassem em um ECOS móvel - "A qualidade do trabalho depende muito disso, e ter acesso a ferramentas de construção de aplicações é essencial para você poder escrever seu código mais rápido, mais eficiente, com maior escalabilidade" (D10). Contudo, alguns informaram que inicialmente têm dificuldade em lidar com os recursos (D11, D16) 
disponibilizados na plataforma, levando algum tempo para se habituarem a estes recursos - "No início eu não tinha tanta noção desses recursos. Eu não conhecia ferramentas, eu não conhecia bibliotecas e esses tipos de coisas" (D16). Porém, para continuarem participando, 17 desenvolvedores consideraram importante os recursos técnicos, sendo que o desenvolvedor que tem participado de um ECOS móvel há mais tempo entre os entrevistados reconheceu que os recursos técnicos têm melhorado consideravelmente ao longo dos últimos anos - "Toda solução e documentação da plataforma que eu desenvolvo está em contínua atualização, a documentação está sempre atualizada e eles estão sempre adicionando novas soluções para os problemas que a gente acaba identificando" (D4). Por fim, a carência dos recursos necessários para o desenvolvedor tende a comprometer o seu desempenho (D5, D8, D17), gerando atrasos e fazendo com que o desenvolvedor tenha que pensar em outras formas de lidar com essas deficiências - "Sempre quando a documentação ou recursos é inexistente, o trabalho acaba sendo prejudicado, por isso que às vezes ocorrem atrasos e bugs" (D8).

\section{(F2) Desempenho do hardware das aplicações}

Inicialmente, os desenvolvedores costumam não prestar muita atenção nas questões de desempenho, sendo essas apontadas como importante ao se iniciar a participação, segundo a opinião de 8 dos entrevistados. Para continuar participando, 18 dos 20 entrevistados reforçam a importância deste fator. O desempenho do hardware dos dispositivos, algumas vezes, torna-se uma preocupação, pois os desenvolvedores precisam lidar com uma grande variação de configurações de hardware (D1, D2, D4, D9, D16, D19) dos usuários finais, a qual é cada vez maior. Entre as razões, fabricantes e modelos novos surgem seguidamente e o desenvolvedor precisa tomar certas precauções para que suas aplicações funcionem nestes diferentes dispositivos - "Eu acho que era mais nivelado quando eu comecei. No início, iPhone e alguns dispositivos Android tinham sua performance praticamente a mesma e hoje se tem desde celulares muito potentes até celulares com pouquíssimos recursos [de hardware]" (D1). Contudo, quando conseguem lidar com estes problemas, os desenvolvedores aumentam suas oportunidades de mercado (D3, D8), uma vez que um desenvolvedor consegue alcançar públicos maiores dentro da mesma plataforma a partir de adaptações em sua aplicação - "Quantos mais dispositivos executarem a sua aplicação, mais pessoas vão conseguir utilizar. Deve-se levar em conta que o cliente consiga executar a aplicação no hardware que ele possuir, para que ele não precise comprar outro dispositivo apenas para essa aplicação” (D3).

\section{(F3) Plataforma facilmente configurável}

A configuração fácil da plataforma foi considerada importante por 12 desenvolvedores para que comecem a participar em um MSECO. Porém, o desenvolvedor apresenta dificuldades ao começar (D4, D6, D8, D19) logo que escolhe uma plataforma, por não possuir o conhecimento necessário para configurar o ambiente de desenvolvimento de forma que se torne mais agradável e produtivo possível - "Eu adquiri o conhecimento de como configurar uma plataforma, então não é algo que eu julgaria tão importante pra mim, mas mais pelo fato de que eu já passei por isso mesmo” (D19). Ainda, 14 desenvolvedores consideraram a importância da plataforma ser simples (D2, D3, D16) para continuarem suas participações em um ECOS móvel, tanto quanto a configuração "Quanto mais fácil seja a plataforma desde o início quando você esta desenvolvimento de aplicações, melhor" (D3). 


\section{(F4) Padrões de interface e aparência das aplicações}

Inicialmente, a importância dos padrões de interface é reconhecida por 14 dos 20 desenvolvedores entrevistados, sendo que alguns inicialmente focavam na funcionalidade e davam menos atenção para a interface das aplicações - "Atualmente a gente se preocupa mais com isso, antigamente não era uma preocupação nossa, a preocupação era a funcionalidade, existiam poucas aplicações” (D7). Além disso, 17 dos 20 desenvolvedores consideram isso importante, e denotam maior foco na aparência das aplicações.

Em uma perspectiva técnica, os recursos técnicos oferecidos pela plataforma são bem recebidos pelo desenvolvedor (D4, D5, D6), pois, facilitam suas atividades quanto à composição de uma interface para suas aplicações, fazendo com que ele tenha mais tempo para outras atividades - "Você não precisar refazer código, já tendo os recursos de interface fica muito mais fácil e simples, desde que esses recursos de interface sejam bons para o cliente, e que se consiga utilizá-los de uma maneira fácil” (D6). Além disso, a existência de padrões de interface oferecidos pela plataforma auxilia o desenvolvedor (D3, D4) a atingir os padrões recomendados pela plataforma, fazendo com que suas aplicações sejam mais padronizadas para os usuários finais e dentro do que se utiliza no mercado "Deixar nos padrões, para ficar uma aparência bonita, uma aplicação que vai chamar a atenção e que vai parecer profissional" (D3).

Além disso, o desenvolvedor deseja agradar os usuários (D2, D11, D16), pois quanto mais agradável for uma aplicação, mais ela vai atrair ou manter os usuários utilizando-na, e potencialmente mais clientes dos serviços embutidos na aplicação surgem - "A gente desenvolve aplicações pensando no cliente, tem que ser uma experiência agradável" (D16). Além disso, a interface de uma aplicação pode ser um diferencial dos concorrentes no mercado (D1, D9), uma vez que mesmo respeitando os padrões e utilizando os recursos oferecidos pela plataforma, o desenvolvedor pode utilizar de sua criatividade e otimizar a experiência de seus usuários - "Isso tornou-se uma forma de diferenciar sua aplicação de outras aplicações, implementando coisas diferentes, mas acredito também que deve-se levar em consideração as questões de usabilidade, não inventando algo completamente fora dos padrões" (D1).

\section{(F5) Segurança dos dados}

A segurança é considerada um fator importante para se começar em um ECOS móvel, segundo a opinião de 15 dos desenvolvedores entrevistados, além de ser importante para continuar, conforme 18 desenvolvedores, o que demonstra a importância deste fator desde o início, para a rotina e para a experiência dos desenvolvedores. A segurança dos dados é uma questão técnica fundamental para os desenvolvedores por se tratar de uma grande preocupação, e, por sua vez, os desenvolvedores reconhecem a importância (D1, D11, D18) de terem esse cuidado especial com a segurança de suas aplicações "Segurança é uma coisa essencial, por mais simples que sejam os dados que você esteja lidando, você sempre pode fazer alguma coisa" (D18). Isso pois os desenvolvedores lidam em suas aplicações com dados sensíveis de seus usuários (D4, D5, D9, D19), que não podem ser expostos ao público - "Deve-se assegurar os dados do próprio cliente, você tem que mostrar para o cliente que criou uma aplicação segura, que ele pode usá-la sem nenhum risco (os dados serem roubados, por exemplo)" (D5). Além disso, os desenvolvedores possuem certo receio da responsabilidade pela segurança (D3, D8, D15) destes 
dados tão importantes aos seus usuários. Contudo, dificilmente eles investigam a fundo a segurança, mas confiam nos recursos da plataforma selecionada - "Seria mais o caso de login e senha a nossa segurança e eu nunca fui atrás pra ter, tipo, uma transmissão criptografada desses dados" (D3).

\section{(F6) Acessibilidade e suporte a diferentes perfis de usuários}

A acessibilidade e o suporte a diferentes perfis de usuário são considerados menos importantes quando um desenvolvedor começa a participar de um ECOS móvel (apenas 4 desenvolvedores apontaram). Apesar de se tornarem mais importantes ao longo do tempo, essas questões continuam sendo pouco trabalhadas, consideradas importantes para 10 dos desenvolvedores entrevistados quando se pensa em continuar participando de um ECOS móvel. A realidade é que alguns desenvolvedores não sentem necessidade (D1, D3, D7), por nunca terem que criar uma aplicação com tais características - "Nunca pensei assim, em desenvolver uma aplicação tão modal” (D1).

Todavia, em seus projetos de aplicações, os desenvolvedores costumam possuir um público-alvo estipulado (D6, D14, D15) pelos seus superiores hierárquicos nas empresas ou organizações que trabalham - "Geralmente as aplicações que a gente faz têm nicho específico. Temos que desenvolver e ir pensando neste público-alvo" (D15). Contudo, mesmo dentro de públicos-alvo bem estipulados, um desenvolvedor deseja alcançar o máximo de clientes (D5, D11, D16) possível, pois quer atender o máximo de usuários naquele nicho, atraindo assim possíveis investimentos, inclusive em nichos mais específicos como as necessidades de acessibilidade - "Todo mundo tem que ser capaz de usar a aplicação. O alcance de mais pessoas possível, mas é difícil saber como começar" (D16).

\section{Discussão}

Para um desenvolvedor de tecnologias móveis, em alguns casos, os recursos técnicos (F1) da plataforma se tornam critério de decisão na escolha de um ECOS. Da mesma forma, o desenvolvedor percebe a importância de conseguir facilmente configurar a plataforma (F3), da segurança dos dados trafegados por suas aplicações (F5), entre outros fatores que não apenas o aproxima de um ECOS móvel como o mantém nele. Todavia, alguns fatores são considerados de menor importância pelos desenvolvedores como, por exemplo, a acessibilidade, conforme apontado por alguns entrevistados, visto que muitos projetos possuem público-alvo restrito e específico.

Existem também alguns desafios os quais um desenvolvedor deve se confrontar. Inicialmente surgem problemas, como adaptar-se aos recursos da plataforma (F1), que muitas vezes são desconhecidos, e aprender a configurar e utilizar a plataforma (F3), o que acaba tomando, no começo da jornada, um considerável tempo. Além disso, outros desafios que surgem são quando faltam recursos técnicos (F1), fazendo com que eles gastem tempo em busca de alternativas, ou quando o mesmo precisa lidar com a variação do hardware dos usuários (F2), bem como a responsabilidade com os dados sensíveis do usuário (F5) - além de problemas técnicos que podem gerar implicações legais.

O foco do desenvolvedor também acaba se alterando com o tempo. Com relação a padrões e interfaces (F4), alguns desenvolvedores afirmaram que tinham foco apenas na funcionalidade quando começavam suas atividades na plataforma. Contudo, com relação à acessibilidade e suporte a diferentes perfis de usuários (F6), alguns desenvolvedores 
afirmam que seu foco está relacionado ao público-alvo que cada aplicação deve atender, o que demonstra a mudanças de foco ao longo de suas experiências.

O suporte que a plataforma presta a um desenvolvedor costuma auxiliá-lo em suas atividades, e os desenvolvedores entrevistados reconhecem que os recursos técnicos (F1) têm melhorado muito ultimamente. Quanto a padrões de interface (F4), eles também se agradam da existência de padrões e de recursos pré-elaborados para poderem modelar o design de suas aplicações.

Os desenvolvedores desejam alcançar mais usuários quanto possível e observam oportunidades de mercado. Por exemplo, desenvolvimento de aplicações de forma que funcionem no máximo de dispositivos possíveis (F2); e elaboração de interfaces mais elegantes que atraiam os usuários (F4) e atendam demandas de usuários com necessidades especiais (F6), para assim atingir o máximo de clientes possível. Os desenvolvedores costumam também prestar atenção nos usuários, tentando agradá-los como, por exemplo, com usabilidade de interface mais amigável (F4), ou demonstrando que o usuário pode confiar seus dados sensíveis às suas aplicações (F5).

\section{Limitações e Dificuldades}

Como todo estudo experimental, algumas limitações e dificuldades foram enfrentadas, entre elas: i) A análise dos dados utilizou o método de card sorting, muito recomendado em caso de agrupamentos. Porém, como a quantidade da dados coletados era considerável, demandando um tempo considerável para sua análise; ii) A heterogeneidade dos entrevistados não foi alta, uma vez que os desenvolvedores convidados para as entrevistas são todos brasileiros. Faz-se necessário um estudo com desenvolvedores de diversos países para que se tenha uma visão mais geral, de modo a confirmar os resultados obtidos; iii) A opinião dos desenvolvedores sofre uma maturação com o passar do tempo e de suas experiências, fazendo com que mesmo o mais experiente dos desenvolvedores entrevistados possa, em algum momento, ter novas conclusões sobre estes fatores; iv) A literatura explorada foi a de ECOS móvel. Entretanto, a literatura mais geral da área de ECOS como um todo pode apresentar mais fatores técnicos que não foram apreciados neste estudo.

\section{Conclusão}

A partir deste estudo, foi possível constatar que os fatores técnicos podem implicar na decisão de um desenvolvedor para participar ou se manter participando de um ECOS móvel. Alguns fatores são melhor entendidos pelos desenvolvedores - e.g., Desempenho do hardware das aplicações (F2), Padrões de interface e aparência das aplicações (F4) e Segurança dos dados (F5) -, e outros menos - e.g., Acessibilidade e suporte a diferentes perfis de usuários (F6) -, pois, pela experiência dos desenvolvedores, houve mais situações que precisassem recorrer a alguns dos fatores mais do que a outros.

Esta pesquisa também contribuiu para esclarecer algumas lacunas identificadas na literatura [de Souza et al. 2016], explorando a dimensão técnica de ECOS de forma a complementar o entendimento de elementos da dimensão social. Além disso, com a participação de desenvolvedores nas entrevistas, pode-se verificar como os fatores técnicos identificados no mapeamento sistemático da literatura afetam suas decisões. Além disso, este estudo pode auxiliar desenvolvedores novatos a escolherem um MSECO 
a partir do entendimento de quais fatores são importantes para os desenvolvedores que já participam a mais tempo em um ecossistema.

Também, esta pesquisa abre possibilidades para trabalhos futuros. Por exemplo, sugere-se investigar em literatura correlata se existem outros fatores técnicos não apreciados neste estudo. Outra possibilidade é a realização de um estudo quantitativo, de forma global, a fim de consultar desenvolvedores de ECOS móvel e buscar a generalização dos resultados obtidos.

\section{Agradecimentos}

Agradecemos ao CNPq (processos 420801/2016-2 e 311256/2018-0) pelo apoio financeiro e a PUCRS pelo subsídio aos assistentes de pesquisa da graduação (Edital BPAPRAIAS 2018). Cleidson de Souza agradece o apoio financeiro da PROPESP/UFPA.

\section{Referências}

Borges, C. S. et al. (2019). Fatores que influenciam desenvolvedores a participar de ecossistemas de software movel. Master's thesis, Pontifícia Universidade Católica do Rio Grande do Sul.

Bosch, J. and Bosch-Sijtsema, P. (2010). From integration to composition: On the impact of software product lines, global development and ecosystems. Journal of Systems and Software, 83(1):67-76.

Campbell, P. and Ahmed, F. (2010). A three-dimensional view of software ecosystems. In Proceedings of the European Conference on Software Architecture: Companion Volume, pages 81-84, Copenhagen, Denmark. ACM.

de Souza, C. R., Figueira Filho, F., Miranda, M., Ferreira, R. P., Treude, C., and Singer, L. (2016). The social side of software platform ecosystems. In Proceedings of the Conference on Human Factors in Computing Systems, pages 3204-3214, San Jose, California. ACM.

Dhungana, D., Groher, I., Schludermann, E., and Biffl, S. (2010). Software ecosystems vs. natural ecosystems: learning from the ingenious mind of nature. In Proceedings of the European Conference on Software Architecture: Companion Volume, pages 96102, Copenhagen, Denmark. ACM.

Fontao, A. d. L., dos Santos, R. P., and Dias-Neto, A. C. (2015). Mobile software ecosystem (mseco): a systematic mapping study. In Proceedings of the IEEE Annual Computer Software and Applications Conference, pages 653-658, Taichung, Taiwan. IEEE.

Mallinson, K. (2015). Smartphone revolution: Technology patenting and licensing fosters innovation, market entry, and exceptional growth. IEEE Consumer Electronics Magazine, 4(2):60-66.

Singer, J., Sim, S. E., and Lethbridge, T. C. (2008). Software engineering data collection for field studies. In Shull, F., Singer, J., and Sjoberg, D. I., editors, Guide to Advanced Empirical Software Engineering, chapter 1, pages 9-34. Springer, London, UK.

Spencer, D. (2009). Card sorting: Designing usable categories. Rosenfeld Media, New York, USA. 